\title{
Concept of Anti Multigroups and its Properties
}

\author{
P. A. Ejegwa \\ Department of Mathematics/Statistics/Computer Science, University of Agriculture, \\ P.M.B. 2373, Makurdi, Nigeria \\ e-mail: ocholohi@gmail.com; ejegwa.augustine@uam.edu.ng
}

\begin{abstract}
The concept of multigroups is an application of multiset to group theory. Multigroup is an algebraic structure of a multiset whose underlying set is a group. The objective of this paper is to introduce the concept of anti multigroups and deduce some related results. We establish that a multiset defined over a group is a multigroup if and only if its complement is an anti multigroup. Finally, some results that connect cuts of multigroups to anti multigroups are considered.
\end{abstract}

\section{Introduction}

The term multisets as buttressed by Knuth [22], was first suggested by N. G. de Bruijn (cf. [6]) in a private communication to D. E. Knuth, as an important generalization of set theory, by relaxing the idea of distinct collection of elements in a set. Multiset theory has been explored in literature [9, 21, 25, 27]. The notion of multisets is a boost to the concept of multigroups via multisets, which generalizes group theory. Nazmul et al. [23] proposed the concept of multigroups in multisets framework and presented a number of results. The notion is parallel to fuzzy groups [24]. A comprehensive account on the concept of multigroups was carried out in [18], and it was established that multigroup via multiset is a generalization of group theory.

The concept of multigroups via multisets has been researched upon since inception.

Received: February 26, 2020; Accepted: April 10, 2020

2010 Mathematics Subject Classification: 03E72, 06D72, 11E57, 19A22.

Keywords and phrases: multisets, multigroups, anti multigroups.

Copyright (C) 2020 P. A. Ejegwa. This is an open access article distributed under the Creative Commons Attribution License, which permits unrestricted use, distribution, and reproduction in any medium, provided the original work is properly cited. 
A number of algebraic properties of order of an element in a multigroup were considered in [3] and some results on multigroups which cut across some homomorphic properties were explored in $[4,10]$. The notions of upper and lower cuts of multigroups were proposed and discussed in details with some number of results in [7], and the notions were extended to homomorphic sense and a number of results were explored [14]. Some group's analogous concepts like normal subgroups, characteristic subgroups, direct product, cosets, factor groups and group actions, etc. have been established in multigroup context $[1,2,8,11-13,15-17,19,20,26]$.

The motivation of this paper is to extend the notion of anti fuzzy groups [5] to multigroups context. In this paper, we propose the notion of anti multigroups and obtain some of its properties. The paper is organized as follows: In Section 2, preliminaries on multisets and multigroups are reviewed. Section 3 introduces anti multigroups with some number of results. Meanwhile, Section 4 draws conclusion to the paper and suggests areas of future works.

\section{Preliminaries}

In this section, we review some existing definitions and results for the sake of completeness and reference.

Definition 2.1. [27] Let $X$ be a set. A multiset $A$ over $X$ is just a pair $\left\langle X, C_{A}\right\rangle$, where

$$
C_{A}: X \rightarrow \mathcal{N}=\{0,1,2, \ldots\}
$$

is a function, such that for $x \in X$ implies $A(x)$ is a cardinal and $A(x)=C_{A}(x)>0$, where $C_{A}(x)$ denoted the number of times an object $x$ occur in $A$. Whenever $C_{A}(x)=0$, implies $x \notin X$.

Any ordinary set $B$ is actually a multiset $\left\langle B, \chi_{B}\right\rangle$, where $\chi_{B}$ is its characteristic function. The set $X$ is called the ground or generic set of the class of all multisets containing objects from $X$.

Take $X$ to be the set from which multisets are constructed. The multiset $X^{n}$ is the set of all multisets of $X$ such that no element occurs more than $n$ times. Likewise, the multiset $X^{\infty}$ is the set of all multisets of $X$ such that there is no limit on the number of 
occurrences of an element. We denote the set of all multisets over $X$ by $M S(X)$. Our interest is on $\operatorname{MS}(X)$ that is contained in $X^{n}$.

For example, a multiset $A=[a, a, b, b, c, c, c]$ of $X=\{a, b, c\}$ can be represented as $A=\left[a^{2}, b^{2}, c^{3}\right]$. Other forms of multiset representations can be found in literature.

Definition 2.2. [21] Let $X$ be a nonempty set and $X^{n}$ be the multiset space defined over $X$. Then, for any $A \in M S(X) \subseteq X^{n}$, the complement of $A$ in $X^{n}$ denoted by $A^{c}$ is a multiset such that $\forall x \in X$,

$$
C_{A^{c}}(x)=n-C_{A}(x) .
$$

Henceforth, whenever we write $M S(X)$ implies the set of all multisets over $X$ drawn from the multiset space $X^{n}$.

Definition 2.3. [27] Let $A, B \in M S(X)$. Then, $A$ is called a submultiset of $B$ written as $A \subseteq B$ if $C_{A}(x) \leq C_{B}(x) \forall x \in X$. Also, if $A \subseteq B$ and $A \neq B$, then $A$ is called a proper submultiset of $B$ and denoted as $A \subset B$. A multiset is called the parent in relation to its submultiset.

Definition 2.4. [25] Let $A, B \in M S(X)$. Then, the intersection, union and sum of $A$ and $B$, denoted by $A \cap B, A \cup B$ and $A+B$, respectively, are defined by the rules that for any object $x \in X$,

(i) $C_{A \cap B}(x)=C_{A}(x) \wedge C_{B}(x)$,

(ii) $C_{A \cup B}(x)=C_{A}(x) \vee C_{B}(x)$,

(iii) $C_{A+B}(x)=C_{A}(x)+C_{B}(x)$,

where $\wedge$ and $\vee$ denote minimum and maximum, respectively.

Definition 2.5. [25] Let $A, B \in M S(X)$. Then, $A$ and $B$ are comparable to each other if and only if $A \subseteq B$ or $B \subseteq A$, and $A=B$ if and only if $C_{A}(x)=C_{B}(x)$ $\forall x \in X$.

Definition 2.6. [15] Let $X$ be a group. A multiset $A$ over $X$ is called a multigroupoid 
of $X$ if for all $x, y \in X$,

$$
C_{A}(x y) \geq C_{A}(x) \wedge C_{A}(y)
$$

where $C_{A}$ denotes count function of $A$ from $X$ into a natural number $\mathbb{N}$.

Definition 2.7. $[15,23]$ Let $X$ be a group. A multiset $A$ of $X$ is said to be a multigroup of $X$ if it satisfies the following two conditions:

(i) $A$ is a multigroupoid of $X$,

(ii) $C_{A}\left(x^{-1}\right)=C_{A}(x) \forall x \in X$.

The set of all multigroups of $X$ is denoted by $M G(X)$.

It can be easily verified that if $A$ is a multigroup of $X$, then

$$
C_{A}(e)=\bigvee_{x \in X} C_{A}(x) \forall x \in X,
$$

that is, $C_{A}(e)$ is the tip of $A$, where $e$ is the identity element of $X$.

Remark 2.1. [23] Let $X$ be a group and $A$ be a multiset over $X$. If

$$
C_{A}\left(x y^{-1}\right) \geq C_{A}(x) \wedge C_{A}(y)
$$

for all $x, y \in X$, then $A$ is called a multigroup of $X$.

Definition 2.8. [15] Let $A \in M G(X)$. A submultiset $B$ of $A$ is called a submultigroup of $A$ denoted by $B \sqsubseteq A$ if $B$ is a multigroup. A submultigroup $B$ of $A$ is a proper submultigroup denoted by $B \sqsubset A$, if $B \sqsubseteq A$ and $A \neq B$.

Definition 2.9. [7] Let $A \in M G(X)$. Then, the sets $A_{[n]}$ and $A_{(n)}$ defined by

$$
A_{[n]}=\left\{x \in X \mid C_{A}(x) \geq n, n \in \mathbb{N}\right\}
$$

and

$$
A_{(n)}=\left\{x \in X \mid C_{A}(x)>n, n \in \mathbb{N}\right\}
$$

are called the strong and weak upper cuts of $A$. Cleary, $A_{(n)} \subseteq A_{[n]}$. 
Theorem 2.1. [7] Let $A \in M G(X)$. Then $A_{[n]}, n \in \mathbb{N}$ is a subgroup of $X$ for $n \leq C_{A}(e)$.

Definition 2.10. [23] The inverse of an element $x \in X$ in a multigroup $A$ of $X$ is defined by

$$
C_{A}\left(x^{-1}\right)=C_{A^{-1}}(x) \forall x \in X .
$$

It is deducible that, $C_{A^{-1}}(x)=C_{A}(x)=C_{\left(A^{-1}\right)^{-1}}(x)$.

\section{Anti Multigroups and Some Properties}

This section presents anti multigroup as a multigroup in reverse order. We denote a group by $X$ unless otherwise stated.

\subsection{Concept of anti multigroups}

Here, we define anti multigroup and discuss some of its properties.

Definition 3.1. Suppose $X$ is a groupoid. Then, a multiset $A$ of $X$ is called an anti multigroupoid of $X$ if

$$
C_{A}(x y) \leq C_{A}(x) \vee C_{A}(y) \forall x, y \in X .
$$

Definition 3.2. A multiset $A$ of $X$ is called an anti multigroup of $X$ if the following conditions hold:

(i) $C_{A}(x y) \leq C_{A}(x) \vee C_{A}(y) \forall x, y \in X$.

(ii) $C_{A}\left(x^{-1}\right) \leq C_{A}(x) \forall x \in X$.

We denote the set of all anti multigroups of $X$ by $A M G(X)$.

Example 3.1. Let $X=\{e, a, b, c\}$ be a group such that

$$
a b=c, a c=b, b c=a, a^{2}=b^{2}=c^{2}=e .
$$

Then, the multiset $A=\left\{e^{2}, a^{5}, b^{4}, c^{5}\right\}$ is an anti multigroup of $X$.

Proposition 3.1. If $A$ is an anti multigroup of $X$, then the following hold: 
(i) $C_{A}\left(x^{-1}\right)=C_{A}(x) \forall x \in X$.

(ii) $C_{A}(e) \leq C_{A}(x) \forall x \in X$, where $e$ is the identity element of $X$.

(iii) $C_{A}\left(x^{n}\right) \leq C_{A}(x) \forall x \in X, n \in \mathbb{N}$.

Proof. We present the verifications of (i) to (iii) as below.

(i) By Definition 3.2, $C_{A}\left(x^{-1}\right) \leq C_{A}(x) \forall x \in X$. Also,

$$
C_{A}(x)=C_{A}\left(\left(x^{-1}\right)^{-1}\right) \leq C_{A}\left(x^{-1}\right) .
$$

This completes the proof of (i).

(ii) Suppose $x \in X$. Certainly, $x x^{-1}=e$. Thus,

$$
\begin{aligned}
C_{A}(e)=C_{A}\left(x x^{-1}\right) & \leq C_{A}(x) \vee C_{A}(x) \\
& =C_{A}(x) .
\end{aligned}
$$

Hence $C_{A}(e) \leq C_{A}(x) \forall x \in X$.

(iii) For $n \in \mathbb{N}$, we have

$$
\begin{aligned}
C_{A}\left(x^{n}\right) & \leq C_{A}\left(x^{n-1}\right) \vee C_{A}(x) \\
& \leq C_{A}\left(x^{n-2}\right) \vee C_{A}(x) \vee C_{A}(x) \\
& \leq C_{A}(x) \vee C_{A}(x) \vee \ldots \vee C_{A}(x) \\
& =C_{A}(x) \forall x \in X .
\end{aligned}
$$

Proposition 3.2. If $A$ and $B$ are anti multigroups of $X$, then $A \cap B$ is an anti multigroup of $X$.

Proof. Let $x, y \in X$. We have

$$
\begin{aligned}
C_{A \cap B}\left(x y^{-1}\right) & =C_{A}\left(x y^{-1}\right) \wedge C_{B}\left(x y^{-1}\right) \\
& \leq\left[C_{A}(x) \vee C_{A}(y)\right] \wedge\left[C_{B}(x) \vee C_{B}(y)\right] \\
& =\left[C_{A}(x) \wedge C_{B}(x)\right] \vee\left[C_{A}(y) \wedge C_{B}(y)\right]
\end{aligned}
$$




$$
=C_{A \cap B}(x) \vee C_{A \cap B}(y) .
$$

Hence the result.

Corollary 3.1. If $\left\{A_{i}\right\}_{i \in I}$ is a family of anti multigroups of $X$, then $\bigcap_{i \in I} A_{i} \in A M G(X)$.

Proof. Straightforward from Proposition 3.2.

Remark 3.1. Let $A, B \in A M G(X)$. Then, $A \cup B$ is not an anti multigroup of $X$ except either $A \subseteq B$ or $B \subseteq A$.

Definition 3.3. The family of anti multigroups $\left\{A_{i}\right\}_{i \in I}$ of $X$ is said to have inf/sup assuming chain if either $A_{1} \subseteq A_{2} \subseteq \ldots \subseteq A_{n}$ or $A_{1} \supseteq A_{2} \supseteq \ldots \supseteq A_{n}$, respectively.

Theorem 3.1. Let $\left\{A_{i}\right\}_{i \in I}$ be a family of anti multigroups of $X$. If $\left\{A_{i}\right\}_{i \in I}$ have sup/inf assuming chain, then $\bigcup_{i \in I} A_{i} \in A M G(X)$.

Proof. Let $A=\bigcup_{i \in I} A_{i}$, then $C_{A}(x)=\vee_{i \in I} C_{A_{i}}(x)$. We show that

$$
C_{A}\left(x y^{-1}\right) \leq C_{A}(x) \vee C_{A}(y) \forall x, y \in X .
$$

Let $C_{A}(x)>0, C_{A}(y)>0$, then we have $\vee_{i \in I} C_{A_{i}}(x)>0, \vee_{i \in I} C_{A_{i}}(y)>0$. From the fact that $\left\{A_{i}\right\}_{i \in I}$ possesses sup/inf assuming chain, $\exists i_{0} \in I$ such that $C_{A_{i_{0}}}(x)=$ $\vee_{i \in I} C_{A_{i}}(x)$, and also $\exists j_{0} \in I$ such that $C_{A_{j_{0}}}(x)=\vee_{i \in I} C_{A_{i}}(x)$. Then, we have

Case I: $A_{i_{0}} \subseteq A_{j_{0}}$ or

Case II: $A_{j_{0}} \subseteq A_{i_{0}}$.

By Case I, we get $C_{A_{i_{0}}}(x) \leq C_{A_{j_{0}}}(x)$. And so

$$
\begin{aligned}
C_{A}\left(x y^{-1}\right) & =C_{A_{j_{0}}}\left(x y^{-1}\right) \\
& \leq C_{A_{j_{0}}}(x) \vee C_{A_{j_{0}}}(y) \\
& \leq C_{A_{i_{0}}}(x) \vee C_{A_{i_{0}}}(y)
\end{aligned}
$$




$$
\begin{aligned}
& =\bigvee_{i \in I} C_{A_{i}}(x) \vee \underset{i \in I}{\bigvee} C_{A_{i}}(y) \\
& =C_{A}(x) \vee C_{A}(y) .
\end{aligned}
$$

By Case II, it implies that $C_{A_{j_{0}}}(x) \leq C_{A_{i_{0}}}(x)$. Thus

$$
\begin{aligned}
C_{A}\left(x y^{-1}\right) & =C_{A_{i_{0}}}\left(x y^{-1}\right) \\
& \leq C_{A_{i_{0}}}(x) \vee C_{A_{i_{0}}}(y) \\
& \leq C_{A_{j_{0}}}(x) \vee C_{A_{j_{0}}}(y) \\
& =\bigvee_{i \in I} C_{A_{i}}(x) \vee \bigvee_{i \in I} C_{A_{i}}(y) \\
& =C_{A}(x) \vee C_{A}(y) .
\end{aligned}
$$

The proof is completed.

Theorem 3.2. If $A$ and $B$ are anti multigroups of $X$, then the sum of $A$ and $B$ is an anti multigroup of $X$.

Proof. Let $x, y \in X$. We have

$$
\begin{aligned}
C_{A \oplus B}\left(x y^{-1}\right) & =C_{A}\left(x y^{-1}\right)+C_{B}\left(x y^{-1}\right) \\
& \leq\left[C_{A}(x) \vee C_{A}(y)\right]+\left[C_{B}(x) \vee C_{B}(y)\right] \\
& =\left[C_{A}(x)+C_{B}(x)\right] \vee\left[C_{A}(y)+C_{B}(y)\right] \\
& =C_{A \oplus B}(x) \vee C_{A \oplus B}(y) .
\end{aligned}
$$

Hence $A \oplus B \in A M G(X)$.

Remark 3.2. Let $\left\{A_{i}\right\}_{i \in I} \in A M G(X)$. Then $\sum_{i \in I} A_{i} \in A M G(X)$.

Proposition 3.3. A multiset $A$ is an anti multigroup of $X$ if and only if $C_{A}\left(x y^{-1}\right) \leq C_{A}(x) \vee C_{A}(y) \forall x, y \in X$.

Proof. Assume that $A$ is an anti multigroup of $X$. Then the following conditions hold;

$$
C_{A}(x y) \leq C_{A}(x) \vee C_{A}(y) \forall x, y \in X \text { and } C_{A}\left(x^{-1}\right) \leq C_{A}(x) \forall x \in X .
$$


By combining the conditions, we get

$$
C_{A}\left(x y^{-1}\right) \leq C_{A}(x) \vee C_{A}(y) \forall x, y \in X .
$$

Conversely, suppose the given condition is satisfied. Combining the following facts:

$$
C_{A}(e) \leq C_{A}(x), C_{A}\left(x^{-1}\right)=C_{A}(x) \forall x \in X
$$

and

$$
\begin{aligned}
C_{A}(x y) \leq C_{A}\left(x\left(y^{-1}\right)^{-1}\right) & \leq C_{A}(x) \vee C_{A}\left(y^{-1}\right) \\
& =C_{A}(x) \vee C_{A}(y) \forall x, y \in X,
\end{aligned}
$$

we conclude that $A$ is an anti multigroup of $X$.

Theorem 3.3. If $A$ is an anti multigroupoid of a finite group $X$, then $A$ is an anti multigroup.

Proof. Let $x \in X, x \neq e$. Since $X$ is finite, $x$ has a finite order. Thus $x^{n}=e \Rightarrow x^{-1}=x^{n-1}$. Now using the definition of an anti multigroupoid repeatedly, it follows that

$$
\begin{aligned}
C_{A}\left(x^{-1}\right) & =C_{A}\left(x^{n-1}\right)=C_{A}\left(x^{n-2} x\right) \\
& \leq C_{A}\left(x^{n-2}\right) \vee C_{A}(x) \\
& \leq C_{A}(x) \vee \ldots \vee C_{A}(x) \\
& =C_{A}(x) .
\end{aligned}
$$

Hence the result.

Theorem 3.4. Let $A$ be a multiset of $X$. Then $A \in M G(X)$ if and only if $A^{c} \in A M G(X)$.

Proof. Suppose $A \in M G(X)$. It implies that, $\forall x, y \in X$, we have

$$
\begin{aligned}
& C_{A}\left(x y^{-1}\right) \geq C_{A}(x) \wedge C_{A}(y) \\
\Rightarrow & C_{\left(A^{c}\right)^{c}}\left(x y^{-1}\right) \geq C_{\left(A^{c}\right)^{c}}(x) \wedge C_{\left(A^{c}\right)^{c}}(y)
\end{aligned}
$$




$$
\begin{aligned}
& \Rightarrow 1-C_{A^{c}}\left(x y^{-1}\right) \geq 1-C_{A^{c}}(x) \wedge 1-C_{A^{c}}(y) \\
& \Rightarrow-C_{A^{c}}\left(x y^{-1}\right) \geq-1+\left[1-C_{A^{c}}(x) \wedge 1-C_{A^{c}}(y)\right] \\
& \Rightarrow C_{A^{c}}\left(x y^{-1}\right) \leq 1-\left[1-C_{A^{c}}(x) \wedge 1-C_{A^{c}}(y)\right] \\
& \Rightarrow C_{A^{c}}\left(x y^{-1}\right) \leq C_{A^{c}}(x) \vee C_{A^{c}}(y) .
\end{aligned}
$$

Hence $A^{c} \in A M G(X)$.

Conversely, suppose $A^{c}$ is an anti multigroup of $X$. Then for all $x, y \in Y$, we have

$$
\begin{aligned}
& C_{A^{c}}\left(x y^{-1}\right) \leq C_{A^{c}}(x) \vee C_{A^{c}}(y) \\
\Rightarrow & 1-C_{A}\left(x y^{-1}\right) \leq 1-C_{A}(x) \vee 1-C_{A}(y) \\
\Rightarrow & -C_{A}\left(x y^{-1}\right) \leq-1+\left[1-C_{A}(x) \vee 1-C_{A}(y)\right] \\
\Rightarrow & C_{A}\left(x y^{-1}\right) \geq 1-\left[1-C_{A}(x) \vee 1-C_{A}(y)\right] \\
\Rightarrow & C_{A}\left(x y^{-1}\right) \geq C_{A}(x) \wedge C_{A}(y) .
\end{aligned}
$$

Hence $A \in M G(X)$.

Proposition 3.4. Let $A \in A M G(X)$. If $C_{A}(x)>C_{A}(y)$ for some $x, y \in X$. Then $C_{A}(x y)=C_{A}(x)=C_{A}(y x)$.

Proof. Suppose $C_{A}(x)>C_{A}(y)$ for some $x, y \in X$. Now,

$$
C_{A}(x y) \leq C_{A}(x) \vee C_{A}(y)=C_{A}(x) .
$$

Similarly,

$$
C_{A}(x)=C_{A}\left(x y y^{-1}\right) \leq C_{A}(x y) \vee C_{A}(y)=C_{A}(x y) .
$$

Thus, $C_{A}(x y)=C_{A}(x)$. In the same vein, $C_{A}(y x)=C_{A}(x)$. The result follows.

Proposition 3.5. Let $A \in A M G(X)$. Then $C_{A}\left(x y^{-1}\right)=C_{A}(e)$ if and only if $C_{A}(x)=C_{A}(y)$. 
Proof. Assume that $C_{A}\left(x y^{-1}\right)=C_{A}(e) \forall x, y \in X$, where $e$ is the identity of $X$. Then

$$
\begin{aligned}
C_{A}(x)=C_{A}\left(x\left(y^{-1} y\right)\right) & =C_{A}\left(\left(x y^{-1}\right) y\right) \\
& \leq C_{A}\left(x y^{-1}\right) \vee C_{A}(y) \\
& =C_{A}(y) .
\end{aligned}
$$

Similarly,

$$
\begin{aligned}
C_{A}(y)=C_{A}\left(\left(x^{-1} x\right) y^{-1}\right) & =C_{A}\left(x^{-1}\left(x y^{-1}\right)\right) \\
& \leq C_{A}(x) \vee C_{A}\left(x y^{-1}\right) \\
& \leq C_{A}(x) .
\end{aligned}
$$

Hence $C_{A}(x)=C_{A}(y)$.

Conversely, assume $C_{A}(x)=C_{A}(y) \forall x, y \in X$. Thus, we have

$$
C_{A}\left(x y^{-1}\right)=C_{A}\left(y y^{-1}\right) \Rightarrow C_{A}\left(x y^{-1}\right)=C_{A}(e) .
$$

Proposition 3.6. Let $A \in A M G(X)$. Then $C_{A}(x y)=C_{A}(y) \forall x, y \in X$ if and only if $C_{A}(x)=C_{A}(e)$.

Proof. Suppose $C_{A}(x y)=C_{A}(y) \forall y \in X$. Then by letting $y=e$, we have $C_{A}(x)=C_{A}(e) \forall x \in X$.

Conversely, suppose that $C_{A}(x)=C_{A}(e)$. Then $C_{A}(y) \geq C_{A}(x)$ and so

$$
C_{A}(x y) \leq C_{A}(x) \vee C_{A}(y)=C_{A}(y) .
$$

Also,

$$
\begin{aligned}
C_{A}(y)=C_{A}\left(x^{-1} x y\right) & \leq C_{A}(x) \vee C_{A}(x y) \\
& =C_{A}(x y) .
\end{aligned}
$$

Hence $C_{A}(x y)=C_{A}(y) \forall y \in X$. 
Theorem 3.5. Let $A \in A M G(X)$ and if $x, y \in X$ with $C_{A}(x) \neq C_{A}(y)$, then $C_{A}(x y)=C_{A}(y x)=C_{A}(x) \vee C_{A}(y)$.

Proof. Let $x, y \in X$. Since $C_{A}(x) \neq C_{A}(y)$, it implies that $C_{A}(x)<C_{A}(y)$ or $C_{A}(y)<C_{A}(x)$. Suppose $C_{A}(x)<C_{A}(y)$. Then $C_{A}(x y) \leq C_{A}(y)$ and

$$
\begin{aligned}
C_{A}(y)=C_{A}\left(x^{-1} x y\right) & \leq C_{A}\left(x^{-1}\right) \vee C_{A}(x y) \\
& =C_{A}(x) \vee C_{A}(x y) \\
& =C_{A}(x y) .
\end{aligned}
$$

It follows that

$$
\begin{aligned}
C_{A}(y) \leq C_{A}(x y) & \leq C_{A}(x) \vee C_{A}(y) \\
& =C_{A}(y) .
\end{aligned}
$$

From here, we see that $C_{A}(x y) \leq C_{A}(x) \vee C_{A}(y)$ and $C_{A}(x) \vee C_{A}(y) \leq C_{A}(x y)$ implying that $C_{A}(x y)=C_{A}(x) \vee C_{A}(y)$.

Similarly, suppose $C_{A}(y)<C_{A}(x)$. We have $C_{A}(y x) \leq C_{A}(x)$ and

$$
\begin{aligned}
C_{A}(x)=C_{A}\left(y^{-1} y x\right) & \leq C_{A}\left(y^{-1}\right) \vee C_{A}(y x) \\
& =C_{A}(y) \vee C_{A}(y x) \\
& =C_{A}(y x) .
\end{aligned}
$$

Thus, we get

$$
\begin{aligned}
C_{A}(x) \leq C_{A}(y x) & \leq C_{A}(y) \vee C_{A}(x) \\
& =C_{A}(x) .
\end{aligned}
$$

Clearly, $C_{A}(y x)=C_{A}(y) \vee C_{A}(x)$. Hence the result follows.

Corollary 3.2. If $A$ is an anti multigroup of $X$, then $C_{A}(x y)=C_{A}(x) \vee C_{A}(y)$ $\forall x, y \in X$ with $C_{A}(x) \neq C_{A}(y)$.

Proof. Let $x, y \in X$. Assume that $C_{A}(x)<C_{A}(y)$, then 


$$
C_{A}(x y) \leq C_{A}(x) \vee C_{A}(y)=C_{A}(y) \forall x, y \in X
$$

and

$$
\begin{aligned}
C_{A}(x) \vee C_{A}(y)=C_{A}\left(x^{-1} x y\right) & \leq C_{A}\left(x^{-1}\right) \vee C_{A}(x y) \\
& =C_{A}(x) \vee C_{A}(x y) \\
& =C_{A}(x y) .
\end{aligned}
$$

Thus $C_{A}(x y)=C_{A}(x) \vee C_{A}(y)$.

\subsection{Cuts of anti multigroups}

In this subsection, we propose the idea of cuts of anti multigroups and outline some results.

Definition 3.4. Let $A \in A M G(X)$. Then, the set $\mathbf{A}_{[n]}$ for $n \in \mathbb{N}$ defined by

$$
\mathbf{A}_{[n]}=\left\{x \in X \mid C_{A}(x) \leq n\right\}
$$

is called a $c u t$ of $A$.

Clearly, $\mathbf{A}_{[n]} \cup A_{[n]}=X$ for $n \in \mathbb{N}$.

Proposition 3.7. Let $A$ be an anti multigroup of $X$. Then for $n \in \mathbb{N}$ such that $n \geq C_{A}(e), \mathbf{A}_{[n]}$ is a subgroup of $X$.

Proof. For all $x, y \in \mathbf{A}_{[n]}$, it follows that

$$
C_{A}\left(x y^{-1}\right) \leq\left[C_{A}(x) \vee C_{A}(y)\right] \leq n,
$$

which concludes the proof.

Proposition 3.8. Let $A$ be a multiset of $X$ such that $\mathbf{A}_{[n]}$ is a subgroup of $X \forall n \in \mathbb{N}$ with $n \geq C_{A}(e)$. Then $A$ is an anti multigroup of $X$.

Proof. Let $x, y \in X$ and $C_{A}(x)=n_{1}, C_{A}(y)=n_{2}$. Suppose $n_{2} \geq n_{1}$. Then $x, y \in \mathbf{A}_{[n]}$ so that $x y^{-1} \in \mathbf{A}_{[n]}$. Hence

$$
C_{A}\left(x y^{-1}\right) \leq n_{2}=n_{1} \vee n_{2}=C_{A}(x) \vee C_{A}(y) .
$$




\section{Conclusion}

We have proposed the concept of anti multigroups and deduced some properties of anti multigroups. It was established that a multiset of a group is a multigroup if and only if the complement of the multiset is an anti multigroup. For future research, some analogous results in multigroups could be investigated in anti multigroup setting.

\section{References}

[1] J. A. Awolola, On multiset relations and factor multigroups, South East Asian J. Math. Mathemat. Sci. 15(3) (2019), 1-10.

[2] J. A. Awolola, On cyclic multigroup family, Ratio Mathematica 37 (2019), 61-68.

[3] J. A. Awolola and P. A. Ejegwa, On some algebraic properties of order of an element of a multigroup, Quasi. Related Syst. 25(1) (2017), 21-26.

[4] J. A. Awolola and A. M. Ibrahim, Some results on multigroups, Quasi. Related Syst. 24(2) (2016), 169-177.

[5] R. Biswas, Fuzzy subgroups and anti fuzzy subgroups, Fuzzy Set Syst. 35 (1990), 121124. https://doi.org/10.1016/0165-0114(90)90025-2

[6] N. G. de Bruijn, Denumerations of rooted trees and multisets, Discrete Appl. Math. 6 (1983), 25-33. https://doi.org/10.1016/0166-218X(83)90097-5

[7] P. A. Ejegwa, Upper and lower cuts of multigroups, Prajna Int. J. Math. Sci. Appl. 1(1) (2017), 19-26.

[8] P. A. Ejegwa, A study of multigroup structure and its acting principles on multiset, A Ph.D. Thesis, Ahmadu Bello University, Zaria, Nigeria, 2018.

[9] P. A. Ejegwa, Synopsis of the notions of multisets and fuzzy multisets, Ann. Commun. Math. 2(2) (2019), 101-120.

[10] P. A. Ejegwa and A. M. Ibrahim, Some homomorphic properties of multigroups, Bul. Acad. Ştiinţe Repub. Mold. Mat. 83(1) (2017), 67-76.

[11] P. A. Ejegwa and A. M. Ibrahim, Normal submultigroups and comultisets of a multigroup, Quasi. Related Syst. 25(2) (2017), 231-244.

[12] P. A. Ejegwa and A. M. Ibrahim, Direct product of multigroups and its generalization, Int. J. Math. Combin. 4 (2017), 1-18.

[13] P. A. Ejegwa and A. M. Ibrahim, On comultisets and factor multigroups, Theory Appl. Math. Computer Sci. 7(2) (2017), 124-140. 
[14] P. A. Ejegwa and A. M. Ibrahim, Homomorphism of cuts of multigroups, Gulf J. Math. 6(1) (2018), 61-73.

[15] P. A. Ejegwa and A. M. Ibrahim, Some group's analogous results in multigroup setting, Ann. Fuzzy Math. Inform. 17(3) (2019), 231-245.

https://doi.org/10.30948/afmi.2019.17.3.231

[16] P. A. Ejegwa and A. M. Ibrahim, Some properties of multigroups, Palestine J. Math. 9(1) (2020), 31-47.

[17] Y. Feng and B. O. Onasanya, Multigroups and multicosets, Italian J. Pure Appl. Math. 41 (2019), 251-261.

[18] A. M. Ibrahim and P. A. Ejegwa, A survey on the concept of multigroup theory, $J$. Nigerian Assoc. Math. Physics 38 (2016), 1-8.

[19] A. M. Ibrahim and P. A. Ejegwa, Multigroup actions on multiset, Ann. Fuzzy Math. Inform. 14(5) (2017), 515-526. https://doi.org/10.30948/afmi.2017.14.5.515

[20] A. M. Ibrahim and P. A. Ejegwa, Characteristic submultigroups of a multi-group, Gulf J. Math. 5(4) (2017), 1-8.

[21] S. P. Jena, S. K. Ghosh and B. K. Tripathy, On the theory of bags and lists, Inform. Sci. 132 (2001), 241-254. https://doi.org/10.1016/S0020-0255(01)00066-4

[22] D. Knuth, The Art of Computer Programming, Seminumerical Algorithms, 2nd ed., volume 2, Addison-Wesley, Reading, Massachusetts, 1981.

[23] Sk. Nazmul, P. Majumdar and S. K. Samanta, On multisets and multi-groups, Ann. Fuzzy Math. Inform. 6(3) (2013), 643-656.

[24] A. Rosenfeld, Fuzzy groups, J. Math. Anal. Appl. 35 (1971), 512-517. https://doi.org/10.1016/0022-247X(71)90199-5

[25] D. Singh, A. M. Ibrahim, T. Yohanna and J. N. Singh, An overview of the applications of multisets, Novi Sad J. Math. 37(2) (2007), 73-92.

[26] P. Suma and S. J. John, Multiset approach to algebraic structures, in: Handbook of Research on Emerging Applications of Fuzzy Algebraic Structures, IGI Global Publisher, Hershey, Pennsylvania 17033-1240, USA, 2020, pp. 78-90.

https://doi.org/10.4018/978-1-7998-0190-0.ch005

[27] A. Syropoulos, Mathematics of Multisets, Springer-Verlag Berlin Heidelberg, 2001, pp. 347-358. https://doi.org/10.1007/3-540-45523-X_17 